(1)

CrossMark

\title{
Lung lymphoid neogenesis in cystic fibrosis: a model of adaptive responses to bacteria?
}

\author{
Sophie T. Gohy ${ }^{1,2,3}$, Maha Z. Ladjemi ${ }^{4}$ and Charles Pilette ${ }^{1,2,5}$
}

Affiliations: ${ }^{1}$ Institute of Experimental and Clinical Research - Pole of Pneumology, ENT and Dermatology, Université catholique de Louvain (UCL), Brussels, Belgium. ${ }^{2}$ Dept of Pneumology, Cliniques universitaires St-Luc, Brussels, Belgium. ${ }^{3}$ Cystic fibrosis reference center, Cliniques universitaires St-Luc, Brussels, Belgium. ${ }^{4}$ UMR Inserm U1152, Labex Inflammex, Université Paris 7, Paris Diderot, Paris, France. ${ }^{5}$ Institute for Walloon Excellence in Lifesciences and Biotechnology (WELBIO), Brussels, Belgium.

Correspondence: Charles Pilette, Laboratory of Pulmonary Biology (UCL-IREC-PNEU), Avenue Hippocrate 54 B1-54.04, B-1200 Brussels, Belgium. E-mail: charles.piletteduclouvain.be

@ERSpublications

Frija-Masson and co-workers draw definite conclusions on the role of pathogens for lymphoid neogenesis in the CF lung http://ow.ly/v4iH309MbCs

Cite this article as: Gohy ST, Ladjemi MZ, Pilette C. Lung lymphoid neogenesis in cystic fibrosis: a model of adaptive responses to bacteria?. Eur Respir J 2017; 49: 1700380 [https://doi.org/10.1183/13993003.00380-2017].

Cystic fibrosis and non-cystic fibrosis bronchiectasis share as their main clinical hallmark repeated lung infections by opportunist pathogens. In the normal adult lung, almost no lymphoid tissue is observed, in contrast to fetal and paediatric lungs [1] and in contrast to upper airways [2]. Neogenesis of bronchial-associated lymphoid tissue (BALT), also referred to as induced BALT (iBALT) or ectopic lymphoid follicles, has been observed in several chronic lung diseases, including chronic obstructive pulmonary disease (COPD) [3], lung cancer [4], pulmonary hypertension [5], post-transplant restrictive allograft syndrome [6] or rheumatoid lung [7], as well as possibly, to some extent, in asthma [8]. A distinction should thus be made between aggregates of B-cells without specific reorganisation and lymphoid follicle structures as observed in primary (bone marrow, thymus) and secondary lymphoid organs (lymph nodes, spleen and Peyer's patches). Lymphoid follicles contain mature naïve and memory B-cells, T-cells, dendritic cells and follicular dendritic cells organising in germinal centres and vascularised with lymphatics and high endothelial veinules. Such lymphoid follicles in non-lymphoid organs are called mucosal-associated lymphoid tissue in mucosal tissues and tertiary lymphoid follicles in other organs.

FRIJA-MASSON et al. [9] reveal, in this issue of the European Respiratory Journal, the presence of lymphoid follicles in peribronchial areas of the lungs from patients with cystic fibrosis or with localised bronchiectasis, following careful examination of their structures including B- and T-cells, germinal centres and high-endothelial veinules. In addition, by using an elegant murine model of instillation of microbeads coated with Staphylococcus aureus or Pseudomonas aeruginosa, they were able to recapitulate in these mice the formation of lung lymphoid follicles upon persistent infection. Furthermore, they highlighted in both situations (patients with bronchiectasis and infected mice) increases in IL-17 and the B-cell-attracting chemokine CXCL13 and, to some extent, CXCL12. Accordingly, another recent study reported the presence of lymphoid follicles in the lungs of patients with end-stage cystic fibrosis, localised in peribronchial as well as in parenchymal and perivascular areas [10].

The mechanisms of lymphoid neogenesis remain poorly understood, notably in the lung. It has been shown that the B-cell chemokine CXCL13 is upregulated in COPD and pulmonary hypertension, as well as in 
smoking mice $[5,11]$, presumably produced by stromal fibroblasts and attracting (in areas with abundant reticular fibres and collagen IV) mature B-cells and a subset of follicular T-helper cells which express high levels of its receptor CXCR5. Importantly, CXCL13 is probably induced following lymphotoxin- $\beta$ receptor activation and early upregulation of the IL- $1 \alpha$ pathway, as recently shown following influenza infection of mice [12]. Other B-cell chemokines, such as CXCL12 and CCL19, 20 and 21, probably also contribute to attract circulating CCR6+ CXCR5+ B-cells to lung lymphoid follicles [5, 13], while factors such as B-cell activating factor further promote B-cell survival and immunoglobulin synthesis [14].

The role of lung lymphoid follicles remains debated [15-17], as they can probably be viewed as beneficial or detrimental according to the primum movens, and thereby to the context, of their genesis relating to immune protection against pathogens or autoimmunity against self-antigens. In the model of influenza infection, a protective role of iBALT and secreted antibodies can be demonstrated [18]. The role of bacteria as inducers of lymphoid follicles in the lung has first been reported by DeLventhal et al. [19] following instillation of Haemophilus in the lungs of pigs. This was reproduced by FleIGE et al. [20] upon repeated instillations of $P$. aeruginosa in mice, which induced BALT following IL-17-driven CXCL12, despite the absence of follicular dendritic cells, in contrast to BALT induced by the modified vaccinia virus Ankara. The results of the study reported by FrIJA-MASSON et al. [9] rather suggest that the recruitment/differentiation of these dendritic cells, observed in lymphoid follicles induced by both P. aeruginosa and S. aureus, depends on the persistence of the bacterial trigger rather than the nature of the pathogen. The chronicity is thus a key issue, very relevant to cystic fibrosis lung disease, which is usually characterised even at early stages, by the chronic or repeated presence of opportunistic pathogens in the lung. These infection-induced lymphoid follicles are presumably beneficial, at least in part, by providing adaptive responses to bacteria (e.g. to $P$. aeruginosa) which can be seen in most adult cystic fibrosis patients $[21,22]$ and, in clinical practice, serum anti- $P$. aeruginosa antibodies may help to assess the chronicity of $P$. aeruginosa infection, along with Leed's criteria [23]. In contrast, at the other end of the spectrum, autoantibody production associated with some phenotypes of chronic lung diseases could play a pathogenic role, as reported in pulmonary hypertension [24]. This dichotomic view of infection-versus autoimmunity-related neolymphogenesis is however challenged by recent observations in cystic fibrosis. Although bacterial infection was for long suspected to induce BALT in the cystic fibrosis lung, as nicely confirmed herein using appropriate tools [9], autoantibodies to bactericidal permeability-increasing protein (BPI) as well as to carbamylated proteins are frequently found in patients with cystic fibrosis (up to $80 \%$ of cases) and correlated with worse prognosis in

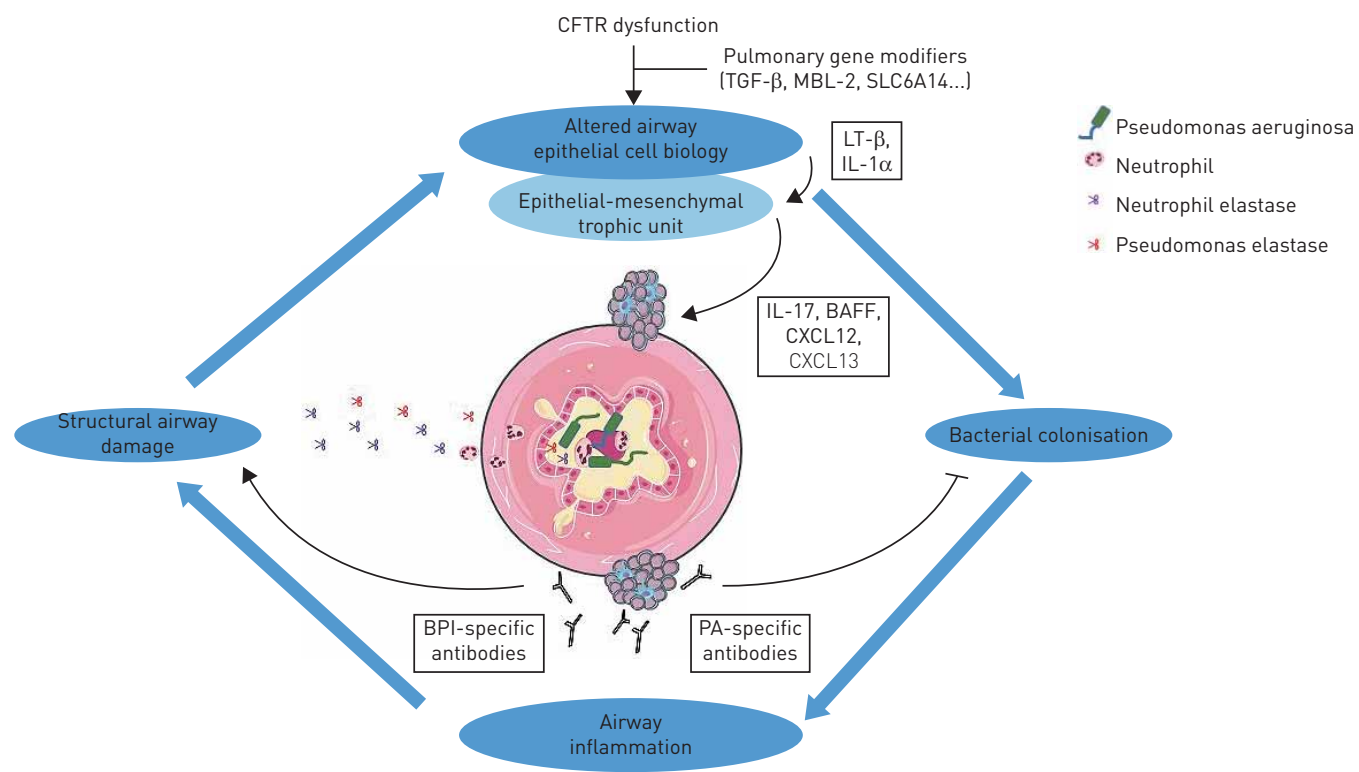

FIGURE 1 Schematic overview of peribronchial lymphoid follicles in the pathophysiology of cystic fibrosis. Upon activation by lymphotoxin (LT)- $\beta$ and interleukin (IL)- $1 \alpha$, airway epithelial and mesenchymal cells secrete chemokines that attract B-cells and contribute to local lymphoid neogenesis. Lymphoid follicles are probably the site of adaptive immunity for the production of anti-Pseudomonas (PA) antibodies as well as anti-bactericidal permeability-increasing protein (BPI) autoantibodies, which are presumably beneficial or detrimental, respectively. Images from Servier Medical Art were used to generate this figure. CFTR: cystic fibrosis transmembrane conductance regulator; TGF- $\beta$; transforming growth factor- $\beta$; MBL-2: mannosebinding lectin 2; SLC6A14: sodium- and chloride-dependent neutral and basic amino acid transporter $\mathrm{B}(0+)_{\text {; }}$ BAFF: B-cell activating factor; CXCL: chemokine (C-X-C motif) ligand. 
terms of mortality, lung function, exacerbations and pan-resistant $P$. aeruginosa [25]. Interestingly, a recent study unravelled that $P$. aeruginosa-mediated formation of neutrophil extracellular traps results in BPI cleavage by $P$. aeruginosa elastase [26], suggesting a novel mechanism of autoimmunity and indicating that complex relationships link infection, (neutrophilic) inflammation and autoimmunity in the cystic fibrosis lung (figure 1).

The next question we need to answer to restore the delicate balance for mucosal tolerance and immunity in the lung, is how can we intervene in cystic fibrosis to transform lymphoid follicles into true friends by switching off the production of autoantibodies and promoting the protective response by anti-bacterial antibodies? Besides sterile pathogenic mechanisms operating in cystic fibrosis [27], addressing these issues may pave the way for current and future studies of the complex interactions between bacteria and airway host tissues in the chronic diseased lung, which probably underlie disease severity, and for which FrijA-Masson et al. [9] have laid an important foundation stone.

\section{References}

1 Pabst R, Gehrke I. Is the bronchus-associated lymphoid tissue (BALT) an integral structure of the lung in normal mammals, including humans? Am J Respir Cell Mol Biol 1990; 3: 131-135.

2 Kato A, Hulse KE, Tan BK, et al. B-lymphocyte lineage cells and the respiratory system. J Allergy Clin Immunol 2013; 131: 933-957.

3 Hogg JC, Chu F, Utokaparch S, et al. The nature of small-airway obstruction in chronic obstructive pulmonary disease. N Engl J Med 2004; 350: 2645-2653.

4 Germain C, Gnjatic S, Tamzalit F, et al. Presence of B cells in tertiary lymphoid structures is associated with a protective immunity in patients with lung cancer. Am J Respir Crit Care Med 2014; 189: 832-844.

5 Perros F, Dorfmuller P, Montani D, et al. Pulmonary lymphoid neogenesis in idiopathic pulmonary arterial hypertension. Am J Respir Crit Care Med 2012; 185: 311-321.

6 Vandermeulen E, Lammertyn E, Verleden SE, et al. Immunological diversity in phenotypes of chronic lung allograft dysfunction: a comprehensive immunohistochemical analysis. Transpl Int 2017; 30: 134-143.

7 Rangel-Moreno J, Hartson L, Navarro C, et al. Inducible bronchus-associated lymphoid tissue (iBALT) in patients with pulmonary complications of rheumatoid arthritis. J Clin Invest 2006; 116: 3183-3194.

8 Elliot JG, Jensen CM, Mutavdzic S, et al. Aggregations of lymphoid cells in the airways of nonsmokers, smokers, and subjects with asthma. Am J Respir Crit Care Med 2004; 169: 712-718.

9 Frija-Masson J, Martin C, Regard L, et al. Bacteria-driven peribronchial lymphoid neogenesis in bronchiectasis and cystic fibrosis. Eur Respir J 2017; 49: 1601873.

10 Lammertyn EJ, Vandermeulen E, Bellon H, et al. End-stage cystic fibrosis lung disease is characterised by a diverse inflammatory pattern: an immunohistochemical analysis. Respir Res 2017; 18: 10.

11 Bracke KR, Verhamme FM, Seys LJ, et al. Role of CXCL13 in cigarette smoke-induced lymphoid follicle formation and chronic obstructive pulmonary disease. Am J Respir Crit Care Med 2013; 188: 343-355.

12 Neyt K, GeurtsvanKessel CH, Deswarte K, et al. Early IL-1 signaling promotes iBALT induction after influenza virus infection. Front Immunol 2016; 7: 312.

13 Neyt K, Lambrecht BN. The role of lung dendritic cell subsets in immunity to respiratory viruses. Immunol Rev 2013; 255: 57-67.

14 Polverino F, Cosio BG, Pons J, et al. B cell-activating factor. An orchestrator of lymphoid follicles in severe chronic obstructive pulmonary disease. Am J Respir Crit Care Med 2015; 192: 695-705.

15 Brusselle GG, Demoor T, Bracke KR, et al. Lymphoid follicles in (very) severe COPD: beneficial or harmful? Eur Respir J 2009; 34: 219-230.

16 Yadava K, Marsland BJ. Lymphoid follicles in chronic lung diseases. Thorax 2013; 68: 597-598.

17 Curtis JL, Freeman CM, Huffnagle GB. "B" for bad, beneficial, or both? Lung lymphoid neogenesis in chronic obstructive pulmonary disease. Am J Respir Crit Care Med 2015; 192: 648-651.

18 Moyron-Quiroz JE, Rangel-Moreno J, Kusser K, et al. Role of inducible bronchus associated lymphoid tissue (iBALT) in respiratory immunity. Nat Med 2004; 10: 927-934.

19 Delventhal S, Hensel A, Petzoldt K, et al. Effects of microbial stimulation on the number, size and activity of bronchus-associated lymphoid tissue (BALT) structures in the pig. Int J Exp Pathol 1992; 73: 351-357.

20 Fleige H, Ravens S, Moschovakis GL, et al. IL-17-induced CXCL12 recruits B cells and induces follicle formation in BALT in the absence of differentiated FDCs. J Exp Med 2014; 211: 643-651.

21 Konstan MW, Hilliard KA, Norvell TM, et al. Bronchoalveolar lavage findings in cystic fibrosis patients with stable, clinically mild lung disease suggest ongoing infection and inflammation. Am J Respir Crit Care Med 1994; 150: $448-454$.

22 Aanaes K, Johansen HK, Poulsen SS, et al. Secretory IgA as a diagnostic tool for Pseudomonas aeruginosa respiratory colonization. J Cyst Fibros 2013; 12: 81-87.

23 Lee TWR, Brownlee KG, Conway SP, et al. Evaluation of a new definition for chronic Pseudomonas aeruginosa infection in cystic fibrosis patients. J Cyst Fibros 2003; 2: 29-34.

24 Colvin KL, Cripe PJ, Ivy DD, et al. Bronchus-associated lymphoid tissue in pulmonary hypertension produces pathologic autoantibodies. Am J Respir Crit Care Med 2013; 188: 1126-1136.

25 Lachenal F, Nkana K, Nove-Josserand R, et al. Prevalence and clinical significance of auto-antibodies in adults with cystic fibrosis. Eur Respir J 2009; 34: 1079-1085.

26 Skopelja S, Hamilton BJ, Jones JD, et al. The role for neutrophil extracellular traps in cystic fibrosis autoimmunity. JCI Insight 2016; 1: e88912.

27 Montgomery ST, Mall MA, Kicic A, et al. Hypoxia and sterile inflammation in cystic fibrosis airways: mechanisms and potential therapies. Eur Respir J 2017; 49: 1600903. 